\title{
Advanced Nursing Practice and Research Contributions to Precision Medicine
}

Janet K. Williams ${ }^{a}$, Maria C. Katapodib ${ }^{\text {, Angela Starkweather }}{ }^{\mathrm{c}}$, Laurie Badzek ${ }^{\mathrm{d}}$, Ann K. Cashion ${ }^{\mathrm{e}}$, Bernice Coleman ${ }^{f}$, Mei R. Fu' ${ }^{g}$, Debra Lyon ${ }^{\mathrm{h}}$, Michael T. Weaver', and Kathleen T. Hickey'

aCorresponding Author: The University of lowa, School of Nursing, 426 College of Nursing Building, lowa City, IA 52246, Phone: 319-335-7046, Email: janet-williams@uiowa.edu

bUniversity of Basel, Institute of Nursing Science, Bernoulli Street 28, Room 113, Basel, Switzerland 4056 Phone: +41 (0) 6126704 30, Email: maria.katapodi@unibas.ch

'University of Connecticut, School of Nursing, Augustus Storrs Hall, 231 Glenbrook Road, Storrs, CT 06269, Phone: 860-486-0592, Email:angela.starkweather@uconn.edu

dUniversity of North Carolina, College of Health and Human Services, $601 \mathrm{~S}$. College Road, Wilmington, NC 28403-5995, Phone: 910-962-7410, Email: Ibadzek@hsc.wvu.edu

eNINR/NIH, 3 Center Drive, Building 3/5E28, Bethesda, MD 20892, Phone: 301-496-0294, Email: ann.cashion@nih.gov

${ }^{f}$ Cedars-Sinai Medical Center Nursing Research and Development, North Tower, Room 2022, 8700 Beverly BLVD, Los Angeles, CA 90048, Phone: 310 423-6178, Email:Bernice.Coleman@cshs.org

9New York University, College of Nursing, 433 First Ave Room 426, New York, NY 10010, Phone: 212998-5314, Email: mf67@nyu.edu

hUniversity of Florida, College of Nursing, PO Box 100197, Gainesville, FL 32610, Phone: 352-273-6335 Email: delyon@ufl.edu

'University of Florida, College of Nursing, PO Box 100197, Gainesville, FL 32610-0197, Phone: 352-2737491, Email: michael.weaver@ufl.edu

jColumbia University, School of Nursing, 617 W. 168 $8^{\text {th }}$ St., Room 356, New York, NY 10032, Phone: $212-$ 305-4944, Email: kth6@cumc.columbia.edu 


\begin{abstract}
Background: Genomic discoveries in the era of precision medicine hold the promise for tailoring healthcare, symptom management, and research efforts including targeting rare and common diseases through the identification and implementation of genomic-based risk assessment, treatment, and management. However, the translation of these discoveries into tangible benefits for the health of individuals, families, and the public is evolving.

Purpose:In this article, members of the Genetics Expert Panel identify opportunities for action to increase advanced practice nursing and research contributions toward improving genomic health for all individuals and populations.

Discussion: Identified opportunities are within the areas of: bolstering genomic focused advanced practice registered nurse practice, research and education efforts; deriving new knowledge about disease biology, risk assessment, treatment efficacy, drug safety and selfmanagement; improving resources and systems that combine genomic information with other healthcare data; and advocating for patient and family benefits and equitable access to genomic healthcare resources.
\end{abstract}


Highlights

- $\quad$ APRNs use genomic knowledge in risk assessment, treatment and management plans.

- Nurse scientists need knowledge of genomic analysis, methods, and applications.

- Nursing leaders can influence the development and implementation of genomic data.

- $\quad$ APRNs provide genomic-based care and advocate for personalized healthcare for all. 


\section{Introduction and Problem Identification}

The promise of personalized medicine, with its focus on individual characteristics, including genetic factors, has inspired the healthcare community to incorporate new and evolving technologies into routine care and optimize their use, while adhering to ethical mandates of clinical practice. This represents a paradigm shift from genetics and genomics regarded as a "special interest addressing rare diseases, to the use of genetic information in all aspects of health care" (Wilson \& Nicholls, 2015, p.10).

The recently announced U.S.A. Precision Medicine Initiative aims at discovering the right treatment, for the right patient, at the right time, as well as discovery of factors contributing to or protecting from common and complex diseases (The White House, 2015). The community of nurse scientists and advanced practice registered nurses (APRN) must be prepared to engage in practice and research that will incorporate genomics and contribute to precision medicine in many diverse areas. Nurses must also advocate for patients' rights regarding recruitment and consent to participating in genomic-related research, as well as use of personal genomic health information for research purposes that is consistent with individual preferences.

Nurses, including ethnic minority nurses, are essential members of the interdisciplinary healthcare team who must also be prepared to use genomic information for guiding the right diagnosis and treatment in clinical practice (Coleman et al., 2014). As a fundamental nursing role, this will entail educating individuals, patients and families regarding the implications of genomics and how genomic findings and future advancements may guide and impact health care treatment, self-management choices, and decision-making. However, it is critical that these opportunities be extended to all including the underserved and minorities, and not limited to those with access, education, and resources to benefit from genomic healthcare. To ensure that personalized medicine will be applicable to all individuals within our diverse American population and reduce health disparities, nurse leaders must continue to engage inclusive 
perspectives when incorporating genomics in research, clinical activities and policy measures. This will also entail facilitating the integration of genomic discoveries into healthcare while maintaining protections for individual and family privacy and health choices.

Genomic discoveries are rapidly advancing from characterizing the molecular structure of genomes and understanding disease biology (Green, Guyer, \& National Human Genome Research Institute, 2011) to translation of discoveries to inform clinical decisions for individuals, families, and communities at risk for conditions with significant genetic components. For example, the advent of genomic testing (e.g., Oncotype $D X^{\circledR}$ ) for various forms of cancer, such as breast, prostate, and colon, has made possible personalized predictions about responsiveness to chemotherapy and the risk of cancer recurrence (Bombard et al., 2015). However, translation of genomic discoveries into healthcare is not uniform and depends partly on evaluation of evidence regarding benefits to patient outcomes from application of genomic information by health care providers (Evans \& Khoury, 2013).

The field of pharmacogenetics, the study of genetic influence on both pharmacokinetics and pharmadynamics, will continue to be a high-impact area for precision medicine. Individuals from ethnically diverse populations, such as African Americans, may particularly benefit from precision medicine discoveries in pharmacogenetics. For example, the use of certain medications such as calcineurin inhibitors following organ transplantation may not achieve therapeutic levels in African Americans (Khush et al., 2015). Thus, healthcare providers, including APRNs must integrate new knowledge of known genetic polymorphism into their care related to "high metabolizer" status that is more common in African Americans (Beermann, Ellis, Sudan, \& Harris, 2014; Dirks, Huth, Yates, \& Meibohm, 2004).

In addition, variability in drug response may not be solely due to the high metabolizer status but may be influenced by other underlying differences in biology (Giarelli, 2015; Laftavi et al., 2013; Mehra, Uber, Scott, \& Park, 2002). Inability to detect and interpret the influence of genetic variants on drug response, including common polymorphisms in the cytochrome P450 
(CYP) isoforms that contribute to differences in drug metabolism, could lead to a patient being incorrectly labeled as noncompliant with medication instructions (Mehra et al., 2002). Therefore, using genomic data in healthcare requires more than genomic discoveries. It also requires addressing how these discoveries contribute to health outcomes for individual patients and implementation of this knowledge across diverse populations and healthcare settings. The Institute of Medicine (IOM) Roundtable on Translating Genomic-Based Research for Health highlights challenges and approaches to address issues of research based evidence, health provider education, and health systems capacities relevant to precision medicine (IOM 2014, 2015a, 2015b), while others (Williams \& Cashion, in press; Williams, Cashion, \& Veenstra, 2015) identify unique contributions of nursing to address current and future genomic challenges.

This paper emphasizes the increase in and emerging importance of APRN and nurse scientist engagement in discoveries and implementation of genomics in healthcare. Four critical areas for action are identified. These are: 1) bolstering genomic focused APRN practice, research and education efforts; 2) deriving new knowledge about disease biology, risk assessment, treatment efficacy, drug safety and self-management; 3) improving use of resources and systems that combine genomic information with other healthcare data; and 4) advocating for patient and family benefits and equitable access to genomic healthcare resources.

\section{Take Away Messages}

The key take away messages for each area are identified here. First, nursing leaders who provide graduate and continuing education for APRNs can build in genomic knowledge and skills, based on APRN competencies, to conduct appropriate assessments and clinical decisions for specific patient populations. These assessments include not only assessments of genomic related risk factors but also issues pertinent to the patient or family that will affect how well the patient and family can manage the condition within the treatment plan. 
Second, discovery of new knowledge to inform the development of precision medicine will require nurse scientists to lead teams in newly developing scientific fields, particularly in methods for designing and conducting implementation research with diverse populations. This will also entail expertise in analysis and interpretation of data spanning across individual genetic tests to genomic sequencing.

Third, nursing leadership within health care institutions and at the national level is necessary to inform the development of information systems that have the capacity to organize, store, and share increasingly larger amounts of omic-based data. Integration of genomic tools within electronic health records is needed to monitor genomic discoveries and update findings that are relevant to clinical practice and further research inquiry that is possible through precision medicine. These advancements must be balanced by adhering to patient privacy and preference regarding use of personal health and medical data.

Fourth, the practice of APRNs is historically rooted in ensuring equitable delivery of healthcare for underserved individuals and families. Through expertise in recognizing how socioeconomic and cultural circumstances influence health and decision-making, APRNs are in an ideal position to demonstrate leadership in advocating for individualized genomic health care that does not limit potential access to and benefits from precision medicine. Nurses at all levels of education can play an influential role in informing legislators and healthcare organizations as well as developing policy measures to ensure that genomic healthcare is accessible to all.

\section{APRN Genomic Competency}

APRNs apply genomic knowledge in risk assessment, patient education, genetic testing and interpretation, and clinical patient management (Greco, Tinley, \& Seibert, 2012; Seibert, 2014). However, APRNs and other healthcare providers who had minimal training in genomics during their basic professional education often lack the knowledge to apply clinically meaningful findings from genomic science to individual patients and families. APRNs who lack the knowledge to understand the application of pharmacogenomics within the complex area of 
patient care risk missing opportunities to use genomic discoveries, optimize patients' healthcare, promote patient understanding and facilitate patient/provider engagement. IOM reports (IOM, 2014, 2015a) document the need for ongoing inter-professional genomics education and resources that support clinical decisions in order that healthcare providers and consumers benefit from translation of genomic discoveries into clinical care. The IOM workshop on interprofessional genomic education highlighted the accomplishments in APRN education from the viewpoints of faculty and APRN student (IOM, 2015b). A consensus panel identified essential genetic and genomic competencies required to provide competent APRN nursing care (Greco et al., 2012). Genetic and genomic knowledge that APRNs are expected to incorporate within their practice includes understanding of genetic and genomic influences on health; recommendations for screening, prevention, and treatment choices; assessing and conducting a critical evaluation of genetic, environmental, and epigenetic influences and risks within health and physical assessments; assessing client knowledge, perceptions, and responses to genetic and genomic information; and incorporating genetic and genomic assessment information within the plan of care.

In order to achieve the maximum benefits from genomic discoveries, education of the APRN healthcare workforce must be faster than the rate at which genomic information is coming into healthcare. The American Nurses Credentialing Center (ANCC) certification in Advanced Genetics Nursing is one new mechanism for documenting genomic competencies. Nurses in the United States and worldwide, who demonstrate genomic skills, knowledge, and abilities, have a minimum of 1500 practice hours in a genomic specialty in the past 5 years, and minimum of 30 hours of continuing education in advanced genetic and genomic topics in the past 3 years are eligible to submit a portfolio for expert peer review and certification (AANC, 2015) (www.nursecredentialing.org).

Genetic and genomic competencies and continuing education opportunities are important for preparing educators and clinicians to use this information in healthcare, and 
APRNs must be aware of how these discoveries contribute to health outcomes for individual patients and families. Examples of the necessity of current knowledge of genomic aspects of health are in APRN practice in sports medicine, primary care, and cardiovascular specialty practices. Genomic evidence-based guidelines have been developed for hypertrophic cardiomyopathy ( $\mathrm{HCM})$, which is characterized by a thickening of the heart and if unrecognized and untreated is a leading cause of sudden cardiac death in young asymptomatic individuals. Cascade screening of other family members who otherwise may remain silently at risk is also possible (Musunuru et al., 2015). This type of targeted, high-yield approach to genomic-based screening of individuals recognized by an APRN as being at risk for HCM because of symptoms and findings on a clincial exam could improve early detection and treatment of this potentially fatal condition.

\section{New Knowledge of Genomic Aspects of Precision Medicine}

Nurse scientists engage in research that informs precision medicine on multiple fronts, including but not limited to symptom science, health promotion and wellness, self-management, and end-of-life and palliative care (National Institute of Nursing Research [NINR], 2011). More specifically, nurse scientists investigate complex symptoms or clusters, clarify and standardize how these symptoms can be measured, identify biomarkers, and develop treatments and interventions related to symptoms of illnesses, injuries and conditions (NINR, 2015).

The Evaluation of Genomic Applications in Practice and Prevention (EGAPP) working group (supported by the Centers for Disease Control and Prevention's Office of Public Health Genomics) systematically reviews evidence on genomic applications in clinical practice and produces recommendations regarding their use (EGAPP, 2014). A comprehensive family history and cascade screening provide important evidence to guide the selection of treatment approaches for a growing number of conditions with Tier 1 genetic applications (see Table 1). Resources to guide clinical decisions for all conditions with significant genetic components have 
not yet been developed and evidence to support testing to identify risk for, diagnose, and/or manage some symptoms or diseases remain to be developed (IOM, 2014). 
Table 1. Tier 1 Applications for three genetic conditions*

\begin{tabular}{|c|c|c|c|}
\hline Condition & $\begin{array}{c}\text { Disease Characteristics } \\
\text { and Prevalence }\end{array}$ & $\begin{array}{c}\text { Biomarke } \\
\text { rs }\end{array}$ & Management Recommendations \\
\hline $\begin{array}{l}\text { Familial } \\
\text { hypercholesterolemi } \\
\text { a (FH), } \\
\text { heterozygous } \\
\text { (HeFH) and } \\
\text { homozygous } \\
\text { (HoFH) }\end{array}$ & $\begin{array}{l}\text { Severely elevated Low-density } \\
\text { lipoprotein (LDL) cholesterol } \\
\text { (LDL-C) levels } \\
\text { HeFH: } 20 \text {-fold increase in risk for } \\
\text { coronary heart disease } \\
\text { Untreated men have } 50 \% \text { risk for a } \\
\text { coronary event by age } 50 \text { years; } \\
\text { Untreated women have } 30 \% \text { risk by } \\
\text { age } 60 \text { years } \\
\text { HoFH: Severe coronary heart disease }\end{array}$ & $\begin{array}{l}\text { APOB, } \\
\text { LDLR, and } \\
\text { PCSK9 }\end{array}$ & $\begin{array}{l}\text { The goal of treatment is a reduction greater than } 50 \% \text { in } \\
\text { LDL-C concentration from baseline either with a high- } \\
\text { intensity statin, or ezetimibe monotherapy or a combination } \\
\text { of both. } \\
\text { For details see National Institute for Healthcare } \\
\text { Excellence (NICE), Identification } \\
\text { and management of familial } \\
\text { hypercholesterolaemia, } 2008 \\
\text { https://www.nice.org.uk/guidance/CG71/chapter/1-Guidance }\end{array}$ \\
\hline
\end{tabular}

Hereditary Breast and Ovarian Cancer (HBOC) by mid-20s

Prevalence of HeFH: 1:200-500

individuals

Prevalence of HoFH: 1:160,000-

$1,000,000$ individuals

Prevalence varies in specific

populations

Increased risk for breast, ovarian,

prostate, pancreas, and skin cancer (melanoma)

Lifetime risk for breast cancer $40-80 \%$, often seen by age 50 years

Lifetime risk for ovarian cancer 11-17

$\%$ generally seen at age 30 years or older

Male breast cancer 1-10\%

Prostate cancer Up to $39 \%$

Pancreatic cancer 1-7\%

Prevalence 1:300-500 individuals

\author{
rs \\ $L D L R$, and LDL-C concentration from baseline either with a high- \\ PCSK9 intensity statin, or ezetimibe monotherapy or a combination \\ of both. \\ Excellence (NICE), Identification \\ https://www.nice.org.uk/guidance/CG71/chapter/1-Guidance
}

BRCA1, BRCA2 Additional

markers include PTEN, p53,

Chek2,

STK11, $\mathrm{CDH} 1$
Use family history screening tools to identify women with an increased risk for harmful mutations. Those with positive screening results should receive genetic counseling and if indicated testing. Interventions that may reduce risk for cancer in BRCA mutation carriers include: earlier, more frequent, or intensive cancer screening; use of selective estrogen receptor modulators as risk-reducing medications (e.g., example, tamoxifen or raloxifene); and risk-reducing surgery (for example, mastectomy and/or salpingooophorectomy).

For details see US Preventive Services Task Force (USPSTF), Risk Assessment, Genetic Counseling, and Genetic Testing for BRCA-RelatedCancer in Women, 2013 
Prevalence varies in specific populations

Lynch Syndrome (LS)

\author{
Increased risk for colon, endometrium, \\ ovarian, stomach, small intestine, \\ hepatobillary tract, urinary tract, brain \\ and \\ skin cancers \\ Lifetime risk for colorectal cancer 52 \\ $82 \%$, \\ Mean age of diagnosis 44-61 years \\ Lifetime risk for endometrial cancer 25- \\ $60 \%$, Mean age of diagnosis 48-62 \\ years \\ Lifetime risk for gastric cancer $6-13 \%$, \\ Mean age of diagnosis 56 years \\ Lifetime risk for ovarian cancer 4-12\%, \\ Mean age of diagnosis 42.5 years, with \\ $\sim 30 \%$ diagnosed before age 40 years \\ Mismatch \\ repair \\ (MMR) \\ genes: \\ MLH1, \\ MSH2, \\ MSH6, \\ PMS2, \\ EPCAM
}

http://www.uspreventiveservicestaskforce.org/Page/Topic/re commendation-summary/brca-related-cancer-riskassessment-genetic-counseling-and-genetic-testing

National Cancer Institute (2014a). BRCA1 and BRCA2: Cancer Risk and Genetic Testing. Retrieved from http://www.cancer.gov/about-cancer/causesprevention/genetics/brca-fact-sheet

National Cancer Institute (2014b). Genetics of Breast and Ovarian

Cancer $(P D Q \circledast)$. Retrieved from http://www.cancer.gov/types/breast/hp/breast-ovariangenetics-pdq

Cascade genetic testing in newly diagnosed individuals with colorectal cancer aimed at reducing morbidity and mortality from Lynch syndrome in high-risk relatives, EGAPP Working Group 2009

National Comprehensive Cancer Network (NCCN),

Genetic/Familial High-Risk Assessment: Colorectal, 2014

Evaluation of Genomic Applications in Practice and

Prevention (EGAPP TM)

http://www.tri-

kobe.org/nccn/guideline/colorectal/english/colorectal screen ing.pdf

Prevalence 1:370-440 individuals

${ }^{*}$ Adapted from George, R., Kovak, K., \& Cox, S. L. (2015). Aligning policy to promote cascade genetic screening for prevention and early diagnosis of heritable diseases. Journal of Genetic Counseling, 24, 388-399. DOI 10.1007/s10897-014-9805-5 
Nursing science contributes to the understanding of genomic aspects of prevention, diagnosis, improved clinical care, and health outcomes in individuals and families (Calzone et al., 2010; Genomic Nursing State of the Science Advisory Panel, 2013; NINR, 2011). As more people live longer with chronic disease and associated adverse symptoms, genomic science can help elucidate the mechanisms of symptom variability across stages of disease. This area of nursing research has led to the development and delivery of tailored symptom management interventions and may help patients make informed decisions regarding optimal selection of treatments and better quality of life, for example in self-regulation of exercise in children with cystic fibrosis (Higgins et al., 2013), or monitoring of pain in children and teens with sickle cell disease (Jacob, Duran, Stinson, Lewis, \& Zeltzer, 2013). The Council for the Advancement of Nursing Science (CANS) emphasizes the importance of in-depth knowledge of biologic and behavioral science content, including -omics sciences, as priorities in preparation of $\mathrm{PhD}$ prepared nursing scientists (Henly et al., 2015). Resources that include omics educational opportunities, funding and research resources, and collaborative and interdisciplinary research opportunities are imperative for sustaining genomic nursing research activities that contribute to the evidence for precision medicine and improved health outcomes.

\section{Resources and Systems Capacity}

Implementation of genomic data for improving clinical care will require changes in the healthcare system, including how data are documented and used in existing databases, e.g., state cancer registries, electronic health records, etc. Nurses are key stakeholders in shaping healthcare institution policy regarding collection and use of health information and meaningfully recorded data by all members of the healthcare team (Katapodi et al., 2013). Capacities to store, protect, manage, and share data are being developed, although several scalability, privacy, security, and informed consent issues need to be resolved for data sharing in existing databases (IOM, 2015b). 
Health and medical information currently stored in these databases, and future databases can be critical resources for both practice and research. The cycle that connects scientific discoveries, application in practice, and evaluation of this application leading to revisiting or pursuing new scientific questions will require databases that contain information that is standardized, comprehensive, and within systems that are flexible in responding to emerging needs.

Nurses can use opportunities to shape institutional policies regarding healthcare knowledge systems and have the capacity to participate in "big data science" while at the same time advocating for ethical and societal issues in data sharing. For example, hospital routine data offer potentially important opportunities in terms of genomic and healthcare research because they are available for all patients, can be obtained with an acceptable cost, and could provide standardized information. However, combining genomic with other health data to derive new knowledge about disease biology, treatment efficacy, and drug safety presents several challenges. The healthcare system is largely unprepared to handle large-scale information that is readily available, for example in electronic health records or International Classification of Diseases (ICD) diagnoses. Nurses can take leadership roles in creating and evaluating mechanisms to promote "big data science" that also serves specific patient, ethnic, or cultural populations (Bakken, Stone, \& Larson, 2008; Clancy et al., 2014).

\section{Advocate for Patient and Family Benefits}

Benefits from clinical genetic and genomic discoveries may be especially inaccessible to healthcare consumers who have limited education and financial resources, live in areas where it is difficult to access genetic specialists, or belong to underserved populations who experience healthcare disparities (Jones, Katapodi, \& Lockhart, 2015; Katapodi, Northouse, Milliron, Liu, \& Merajver, 2013). The promise of scientific advancement that promotes health for everyone, as articulated in the Precision Medicine Initiative, includes the premise that patients and those who interact with the healthcare system not only will benefit from scientific advances but will also 
have the opportunity to form true partnerships with the scientific community to engage in research and shape agendas.

This is best illustrated in a case of a college freshman who conducted a genomic analysis of her own cancer, used social media to mobilize others with this cancer in order to "liberate their samples from hospitals where they had their surgery", and set up clinical trials (Terry, 2015, p. 113). It also means that people feel comfortable sharing their own health data, which would lead to mining the data for new information that can be tested and translated back into healthcare (Terry, 2015).

However, the benefits of genomic research for all populations will be limited if fear of discrimination prevents individuals from participating in translational research studies that explore potential real life consequences of using information resulting from genome sequencing (Green, Lautenbach,\& McGuire, 2015). Resources for patient education, advocacy for the underserved, appropriate fit of genomic health information with individual, family, and community preference, and genomic healthcare services that are culturally appropriate and sensitive to varying genomic literacy levels have yet to be a reality.

\section{Recommendations}

The following policy and research opportunities that address one or more of the issues in this paper are identified, as well as steps that nurses can engage in to enhance personalized nursing care for improving genomic healthcare for all individuals:

1. Incorporate and expand genomic education for APRNs as appropriate to support precision medicine and personalized genomic health at all levels of care, across the lifespan and within all practice settings.

2. Endorse CANS Idea Festival proposal to focus PhD training for nurse scientists to enable translation of discovery into practice.

3. Expand doctoral training for nurse scientists and clinicians to prepare an ethnically diverse nursing workforce prepared to implement genomics based health care. 
4. Increase nursing research emphases on promoting health of individuals and families in a wide variety of clinical care and research settings based on personalized genomic health information.

5. Create and evaluate easy to use accessible (i.e., web-based) genomic clinical decision resources and enable access to these resources by APRNs and other healthcare providers across diverse health care settings.

6. Create and evaluate genomic health literacy resources for members of the public that are appropriate for diverse socioeconomic, educational, health literacy, or cultural backgrounds.

7. Actively participate in international, national and institutional committees that formulate policies regarding healthcare knowledge systems.

8. Advocate for recruitment, consent, and access to personal genomic health information that is consistent with each person's preferences.

\section{Acknowledgements}

We would like to thank the American Academy of Nursing Board and the members of the Genetic Expert Panel for their thoughtful insights and review of this manuscript.

The opinions expressed herein are the responsibility of the author (AC) and in no way should be seen as an official recommendation, interpretation, or policy of the National Institutes of Health or the United States Government. 


\section{References}

American Nurses Credentialing Center (ANCC). (2105). Advanced genetics nursing. Retreived from http://nursecredentialing.org/AdvancedGenetics.

Bakken, S., Stone, P. W., \& Larson, E. L. (2008). A nursing informatics research agenda for 2008-18: Contextual influences and key components. Nursing Outlook, 56(5), 206-214. doi: 10.1016/j.outlook.2008.06.007.

Beermann, K. J., Ellis, M. J., Sudan, D. L., \& Harris, M. T. (2014). Tacrolimus dose requirements in African-American and Caucasian kidney transplant recipients on mycophenolate and prednisone. Clinical Tranplantation, 28(7), 762-677. doi: 10.1111/ctr.12376.

Bombard, Y., Rozmovits, L., Trudeau, M., Leighl, N. B., Deal, K., \& Marshall, D. A. (2015). The value of personalizing medicine: Medical oncologists' views on gene expression profiling in breast cancer treatment. Oncologist, 2(4), 351-356. doi: 10.1634/theoncologist.20140268.

Calzone, K. A., Cashion, A., Feetham, S., Jenkins, J., Prows, C. A., Williams, J. K., \& Wung, SF. (2010). Nurses transforming health care using genetics and genomics. Nursing Outlook, 58(1), 26-35. doi: 10.1016/j.outlook.2009.05.001.

Clancy, T. R., Bowles, K. H., Gelinas, L., Androwich I., Delaney, C., Matney, S., . . Westra, B. (2014). A call to action: Engage in big data science. Nursing Outlook, 62(1), 64-65. doi: 10.1016/j.outlook.2013.12.006.

Coleman, B., Calzone, K. A., Jenkins, J., Paniagua, C., Rivera, R., Hong, O., . . Bonham. V. (2014). Multi-ethnic minority nurses' knowledge and practice of genetics and genomics. Journal of Nursing Scholarship, 46(4), 235-244. doi: 10.1111/jnu.12083.

Dirks, N. L., Huth, B., Yates, C. R., \& Meibohm, B. (2004). Pharmacokinetics of immunosuppressants: A perspective on ethnic differences. International Journal of Clinical Pharmacology, Therapy and Toxicology, 42(12), 701-718. 
Evaluation of Genomic Applications in Practice and Prevention (EGAPP) Working Group. (2014). The EGAPP initiative: Lessons learned. Genetics in Medicine, 16(3), 217-224. doi: 10.1038/gim.2013.110

Evans, J. P., \& Khoury, M. J. (2013). The arrival of genomic medicine to the clinic is only the beginning of the journey. Genetics in Medicine 15(4), 268-269. doi: 10.1038/gim.2012.133.

Giarelli E. (2015). Integrating pharamacogeomics into Healthcare. In D. H. Lea, D. J. Cheek, D. Brazeau, \& G. Brazeau (Eds.), Mastering pharmacogenomics: A nurse's handbook for success (pp. 59-78). Indianapolis: Sigma Thea Tau International.

Genomic Nursing State of the Science Advisory Panel, Calzone KA, Jenkins J, Bakos AD, Cashion AK, Donaldson N, . . Webb JA. (2013). A blueprint for genomic nursing science. Journal of Nursing Scholarship, 45(1), 96-104. doi: 10.1111/jnu.12007.

George, R., Kovak, K., \& Cox, S. L. (2015). Aligning policy to promote cascade genetic screening for prevention and early diagnosis of heritable diseases. Journal of Genetic Counseling, 24(3), 388-399. doi 10.1007/s10897-014-9805-5.

Greco, K. E., Tinley, S., \& Seibert, D. (2012). Essential genetic and genomic competenciesfor nurses with graduate degrees. Silver Spring, MD: American Nurses Association and International Society of Nurses in Genetics.

Green, E. D., Guyer, M. S., \& National Human Genome Research Institute. (2011). Charting a course for genomic medicine from base pairs to bedside. Nature, 470, 204-213. doi:10.1038/nature09764

Green, R. C., Lautenbach, D., \& McGuire, A. L. (2015). GINA, genetic discrimination, and genomic medicine. The New England Journal of Medicine 372(5), 397-399. doi: 10.1056/NEJMp1404776.

Henly, S. J., McCarthy, D. O., Wyman, J. F., Heitkemper, M. M., Redeker, N. S., Titler, M. G., . . Dunbar-Jacob, J. (2015). Emerging areas of science: Recommendations for nursing 
science education from the Council for the Advancement of Nursing Science Idea Festival. Nursing Outlook, 63(4), 398-407. doi: 10.1016/j.outlook.2015.04.007.

Higgins, L. W., Robertson, R. J., Kelsey, S. F., Olson, M. B., Hoffman, L. A., Rebovich, P. J., . . . Orenstein, D. M. (2013). Exercise intensity self-regulation using the OMNI scale in children with cystic fibrosis. Pediatric Pulmonology, 48(5), 497-505. doi:

10.1002/ppul.22639.

Institute of Medicine (IOM). (2014). Assessing genomic sequencing information for health care decision making: Workshop summary. Washington DC: The National Academies Press. Institute of Medicine (IOM). (2015a). Improving genetics education in graduate and continuing health professional education: Workshop summary. Washington DC: The National Academies Press.

Institute of Medicine (IOM). (2015b). Genetics-enabled learning health care systems: Gathering and using genomic information to improve patient care and research: Workshop summary. Washington DC: The National Academies Press.

Jacob, E., Duran, J., Stinson, J., Lewis, M., \& Zeltzer, L. (2013). Remoted monitoring of pain and symptoms using wireless technology in children and adolescents with sickle cell disease. Journal of the American Association of Nurse Practitioners, 25(1), 42-54. doi: 10.111/j.1745-7599.2012.00754.x.

Jones, T. P., Katapodi, M. C., \& Lockhart, J. (2015). Factors influencing breast cancer screening and risk assessment among young African American women: An integrative review of the literature. Journal of American Association of Nurse Practitioners, 27(9), 521-529. doi: $10.1002 / 2327-6924.12223$.

Katapodi, M. C., Northouse, L. L., Milliron, K. J., Liu, G., \& Merajver, S. D. (2013). Individual and family characteristics associated with BRCA1/2 testing in high-risk families. Psychooncology, 22(6), 1336-1343. doi: 10.1002/pon.3139. 
Katapodi, M. C., Northouse, L. L., Schafenacker, A. M., Duquette, D., Duffy, S. A., Ronis, D., .. . Copeland. (2013). Using a state cancer registry to recruit young breast cancer survivors and high-risk relatives: A protocol of a randomized trial testing the efficacy of a targeted versus a tailored intervention to increase breast cancer screening. BMC Cancer, 13, 97. doi: 10.1186/1471-2407-13-97.

Khush, K. K., Pham, M. X., Teuteberg, J. J., Kfoury, A. G., Deng, M. C., Kao, A., . . Valantine H. A. (2015). Gene expression profiling to study racial differences after heart transplantation. Journal of Heart and Lung Transplantation, 34(7), 970-977. doi: 10.1016/j.healun.2015.01.987.

Laftavi, M. R., Pankewycz, O., Patel, S., Nader, N., Kohli, R., Feng, L., . . Dayton, M. (2013). African American renal transplant recipients (RTR) require higher tacrolimus doses to achieve target levels compared to white RTR: Does Clotrimazole help? Transplantation Proceedings, 45(10), 3498-3501. doi10.1016/j.transproceed.2013.09.010.

Mehra, M., Uber, P., Scott, R., \& Park, M. (2002). Ethnic disparity in clinical outcome after heart transplantation is abrogated using tracolimus and mycophenolate mofetil-based immunosuppression. Transplantation, 74(11), 1568-1573.

Musunuru, K., Hickey, K. T., Al-Khatib, S. M., Delles, C., Fornage, M., Fox, C. S., . . Rosand, J. (2015). Basic concepts and potential applications of genetics and genomics for cardiovascular and stroke clinicians: A scientific statement from the American Heart Association. Circulation: Cardiovascular Genetics, 8(1), 216-242. doi: 10.1161/HCG.0000000000000020.

National Institute of Nursing Research (NINR). (2011). Bringing science to life: NINR strategic plan. Bethesda, MD: National Institutes of Health.

National Institute of Nursing Research (NINR). (2015). Precision medicine and NINR-supported nursing science. Retreived from http://www.ninr.nih.gov/researchandfunding/precisionmedicine. 
Seibert, D. C. (2014). Genomics and nurse practitioner practice. The Nurse Practitioner, 39(10), 18-28. doi: 10.1097/01.NPR.0000453641.13662.03.

Terry, S. F. (2015). Obama's precision medicine initiative. Genetic Testing and Molecular Biomarkers, 19(3), 113-114. doi: 10.1089/gtmb.2015.1563.

The White House. (2015). Fact sheet: President Obama's precision medicine initiative. Retrived from https://www.whitehouse.gov/the-press-office/2015/01/30/fact-sheet-presidentobama-s-precision-medicine-initiative.

Williams, J. K., Cashion, A. K., \& Veenstra, D. L. (2015). Challenges in evaluating nextgeneration sequence data for clinical decisions Nursing Outlook 63(1):48-50. doi: 10.1016/j.outlook,2014.08.007.

Williams, J. K., \& Cashion, A. K. (in press). Using clinical genomics in health care: Strategies to create a prepared workforce. Nursing Outlook.

Wilson, B. J., \& Nicholls, S. G. (2015). The human genome project, and recent advances in personalized genomics. Risk Management and Healthcare Policy, 8, 9-20. doi: 10.2147/RMHP.S58728. 